\title{
Investigation of Separation Method for Gallium Nitride with Internal Modified Layer by Ultrashort Pulsed Laser
}

\author{
Yasuhiro Okamoto*, Motoki Ota*, Akira Okada* \\ (Received Jan. 16, 2019) \\ * Graduate School of Natural Science and Technology, Okayama University, \\ 3-1-1 Tsushimanaka, Kita-ku, Okayama 700-8530, Japan
}

\begin{abstract}
Gallium nitride $(\mathrm{GaN})$ is attractive material for blue light emitting diodes and high-frequency and highpower semiconductors. In general, GaN is sliced by using a wire saw method, although this cutting method has some problems such as thick damaged layer on sliced surface and environmental issue. On the other hand, laser slicing method has the possibility to reduce the kerf loss and the damaged layer compared with the wire saw method. In addition, high quality GaN with low damaged layer can be expected by using an ultrashort pulsed laser irradiation. Therefore, ultrashort pulsed laser was focused inside GaN, and the possibility of its separation by internal modified layer formation was experimentally investigated. GaN is partly decomposed into gallium and nitrogen by ultrashort pulsed laser irradiation, and thin internal modified layer is created by the combination of brittle fracture and decomposition of GaN. The continuous internal modified layer can be obtained by 10 ps pulsed laser irradiation, and GaN can be separated by its internal modified layer.
\end{abstract}

Key words: gallium nitride, separation, ultrashort pulsed laser

\section{INTRODUCTION}

Gallium nitride $(\mathrm{GaN})$ is an attractive material for blue light emitting diode (LED) ${ }^{1)}$, and highfrequency and high-power semiconductors with low energy consumption ${ }^{2-4)}$. In order to obtain GaN substrate, many researchers have investigated some crystal growing methods, such as hydride vapor phase epitaxy (HVPE), metalorganic vapor phase epitaxy (MOPVE), and dislocation elimination by the epitaxial growth with inverse-pyramidal pits (DEEP). HVPE is the method in the first development for crystal growth of $\mathrm{GaN}$ on sapphire substrate $^{5)}$, and aluminum nitride (AIN) was used as a buffer layer in MOPVE to overcome lattice and thermal mismatches between $\mathrm{GaN}$ and sapphire substrate $^{6)}$. DEEP was developed to minimize dislocation area by using cores on gallium arsenide (GaAs) substrate ${ }^{7)}$, ${ }^{8}$. Although some growing methods of single crystalline GaN were proposed, an ingot of GaN is generally grown by heteroepitaxial growth on a substrate. Thus, dislocation area is often generated in lower part of GaN close to a substrate during the crystal growing process, and it leads to reduction of blue light emitting efficiency ${ }^{9), 10)}$. In order to practically apply semiconductor material with high-quality, the dislocation area close to the substrate must be removed, and it is necessary to separate the high dislocation density area. Therefore, high-quality slicing method of GaN is required to obtain bulk GaN with low dislocation density. One of the separation methods is a wire saw, which has been typically used for slicing process of semiconductor materials, although this cutting method has some problems such as thick kerf loss, large damaged layer on sliced surface. Moreover, this slicing method causes an environmental issue, because debris and slurry are mixed in working fluid during the process.

On the other hand, laser slicing method of noncontact processing has the possibility to reduce the kerf loss and the damaged layer compared with the wire saw method. Disco Corporation innovated unique laser slicing method named KABRA ${ }^{11)}$, and the combination of grinding and laser irradiation enables single wafer slicing process. However, this technique can apply only for silicon carbide ( $\mathrm{SiC})$. Yamada et al. reported laser cutting-out process by introducing micro cracks inside a material with ns pulsed laser ${ }^{12)}$, and they also proposed slicing method of single crystalline silicon ( $\mathrm{Si}$ ) and $\mathrm{SiC}$ with ns and fs pulsed lasers. However, a laser slicing of $\mathrm{GaN}$ has not yet been discussed, and there is few report to discuss laser processing inside $\mathrm{GaN}$.

$\mathrm{GaN}$ is highly brittle material, and control of crack propagation is difficult. In addition, GaN has a wide band gap of about $3.4 \mathrm{eV}$, and it is difficult to absorb the laser beam inside GaN with low photon energy ${ }^{13)}$. Tightly focused ultrashort pulsed laser enables nonlinear absorption, and it can be expected that highquality GaN with low damaged layer could be obtained by controlling the cracks with ultrashort pulsed laser irradiation ${ }^{14)}$. Therefore, formation of internal modified layer inside $\mathrm{GaN}$ was experimentally investigated by ultrashort pulsed laser, and the possibility of separation method for $\mathrm{GaN}$ was discussed by using the formation of internal modified layer. 


\section{LASER IRRADIATION METHOD}

Schematic diagram of laser irradiation setup and main experimental conditions are shown in Fig. 1 and Table 1. In order to absorb the energy of laser beam inside GaN, high intensity of laser pulse and transparent wavelength for $\mathrm{GaN}$ are required. Thus, ultrashort pulsed laser of $790 \mathrm{~nm}$ wavelength was employed. Wavelength and pulse repetition rate of ultrashort pulsed laser are $790 \mathrm{~nm}$ and $1.0 \mathrm{kHz}$, respectively. Pulse duration was varied from $0.2 \mathrm{ps}$ to $10 \mathrm{ps}$ by controlling the position of grating. Circular polarized laser beam of $3.5 \mathrm{~mm}$ in diameter was focused inside GaN by using an objective lens with a large numerical aperture (N.A.) of 0.85 . The diameter of laser focusing spot in air was about 5.0 $\mu \mathrm{m}$, but it became a little larger due to its high refractive index of 2.4. GaN of $1.0 \mathrm{~mm}$ thickness without a substrate was used as a specimen, which was fixed on xyz-stage. The top surface of $\mathrm{GaN}$ was finished to a mirror surface as shown in optical microphotograph. Laser scanning experiments were conducted by controlling the feed rate of xyz-stage, and the scanning speed was set to $1.0 \mathrm{~mm} / \mathrm{s}$.

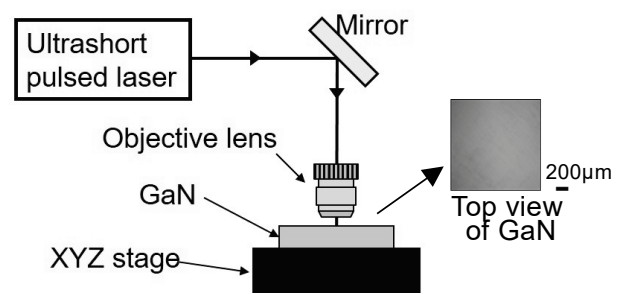

Fig. 1 Experimental setup for laser irradiation

Table 1 Main experiemental conditions

\begin{tabular}{l|l}
\hline Wavelength $\lambda$ & $790 \mathrm{~nm}$ \\
\hline Pulse duration $t_{\mathrm{p}}$ & $0.2 \mathrm{ps}-10 \mathrm{ps}$ \\
\hline Pulse repetition rate $R_{\mathrm{p}}$ & $1.0 \mathrm{kHz}$ \\
\hline Scanning speed $v$ & $1.0 \mathrm{~mm} / \mathrm{s}$ \\
\hline Pulse energy $E$ & $0.85 \mu \mathrm{J}$ \\
\hline Spot diameter $d_{\mathrm{s}}$ & $5.0 \mu \mathrm{m}(\mathrm{In}$ air $)$ \\
\hline Line pitch & $2.0 \mu \mathrm{m}, 5.0 \mu \mathrm{m}$ \\
\hline
\end{tabular}

Figure 2 shows formation method of internal modified layer inside GaN. Laser focusing spot was set at $300 \mu \mathrm{m}$ below the top surface of specimen, and one laser scanning line was created in y-direction. Then, the position of laser beam was moved in $\mathrm{x}$ direction according to the line pitch, and internal modified layer of $2.0 \mathrm{~mm}$ in width was formed at 300 $\mu \mathrm{m}$ below the top surface of GaN. Crystallographic orientation of top surface was C plane (0001), and laser beam was focused inside $\mathrm{GaN}$ from the end side of crystal growth.

In order to observe the cross section of laser scanned specimen, argon ion beam etching equipment was used, and observation areas were prepared by $5 \mathrm{kV}$ of acceleration voltage for 8 hours ion beam irradiation.

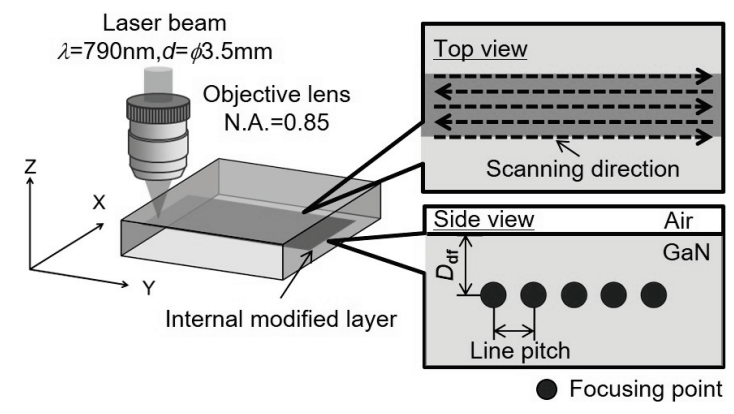

Fig. 2 Formation method of internal modified layer

\section{FORMATION OF INTERNAL MODIFIED LAYER}

Pulse energy of $0.85 \mu \mathrm{J}$ and scanning speed of 1.0 $\mathrm{mm} / \mathrm{s}$ were determined to obtain continues internal modified line in preliminary experiments of single line formation. The pulse energy of $0.80 \mu \mathrm{J}$ was minimum value to form an internal modified line for all pulse durations at the scanning speed of $1.0 \mathrm{~mm} / \mathrm{s}$, and the pulse energy of $0.85 \mu \mathrm{J}$ was selected to create continuous internal modified line, which is important for separation process.

Influence of pulse duration on internal modified layer was investigated at the same pulse energy 0.85 $\mu \mathrm{J}$ and line pitch $2.0 \mu \mathrm{m}$. Figure 3 shows optical microphotographs of laser irradiation area at pulse duration from 0.2 ps to $10 \mathrm{ps}$. Dashed rectangular marks indicate laser irradiation areas. In these photographs, dark area means that illumination light cannot pass through GaN. Non-irradiation area can be recognized as the white part, which indicates there is no defects to disturb the passing of illumination light. In contrast, dark area has defects such as cracks, dislocations and roughed surface, which disturb the passing of illumination light. In cases of $0.2 \mathrm{ps}, 0.8 \mathrm{ps}$ and $3.0 \mathrm{ps}$, dark areas appeared randomly and unevenly, and the high peak laser intensity makes it difficult to control the generation of crack. The cracks propagated widely beyond the absorption region of laser energy, the cracks were largely expanded in perpendicular direction of laser irradiation axis. The crack propagation exceeded the laser irradiation area, and the accurate control of crack propagation is difficult under these conditions.

On the other hand, at $5.0 \mathrm{ps}$ and $10 \mathrm{ps}$, continuous internal modified layer could be obtained, and the formation area of internal modified layer could be controlled inside the laser irradiation area at $10 \mathrm{ps}$. Cross sections of internal modified layer were evaluated at $0.2 \mathrm{ps}$ and $10 \mathrm{ps}$ to distinguish the difference of phenomena. 


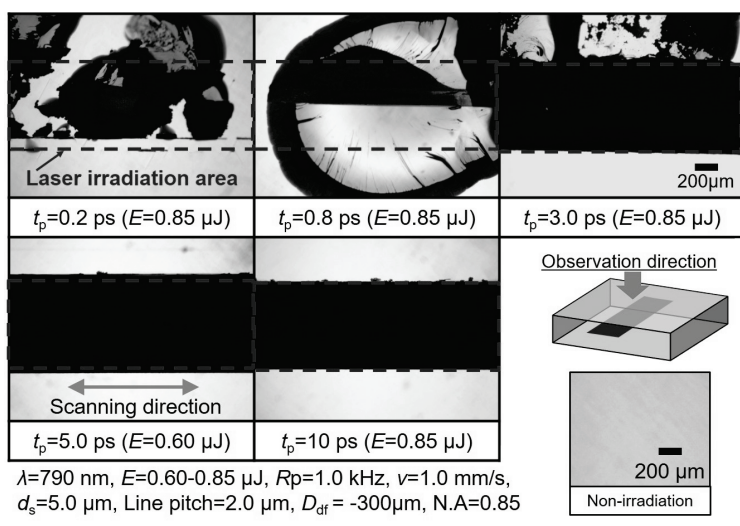

Fig. 3 Optical microphotographs of laser irradiation area for various pulse durations

Figure 4 shows optical microphotographs of top side and scanning electron microscope (SEM) images of cross section around internal modified layer at $0.2 \mathrm{ps}$ and $10 \mathrm{ps}$. The cross section of $0.2 \mathrm{ps}$ is around the area, where there are gray and dark gray zone caused by large cracks at the top view. Some cracks were generated in the laser irradiation areas at both pulse durations, but there was obvious difference in crack propagation state. The length of crack was short at $10 \mathrm{ps}$, and crack generation area could be controlled compared with that at 0.2 ps. 0.2 ps showed one crack disk, but its length was much longer than that at the line pitch of $2.0 \mu \mathrm{m}$. The size of this crack was beyond the absorption region of laser energy. This phenomenon indicates that crack largely propagated in layer direction of crystal growing process. It is difficult to control the size of crack propagation. $10 \mathrm{ps}$ pulse duration would be useful for the formation of internal modified layer inside $\mathrm{GaN}$, since it is considered that continuous and uniform internal modified layers are important for the separation process. These phenomena would be caused by different laser intensity at each pulse duration. Peak power of $0.2 \mathrm{ps}$ pulsed laser is 50 times higher than that of $10 \mathrm{ps}$ pulsed laser under the same pulse energy condition. The high laser intensity leads to a large shockwave inside GaN, and a large crack propagation was suddenly caused at some parts. Because crystal growing of $\mathrm{GaN}$ is mainly performed by layers, and the high intensity of $0.2 \mathrm{ps}$ pulsed laser would increase the crack propagation in the perpendicular direction to laser axis.

On the other hand, small crack was generated by $10 \mathrm{ps}$ pulsed laser due to its small laser intensity, but absorption area of $10 \mathrm{ps}$ pulsed laser was larger than that of $0.2 \mathrm{ps}$ pulsed laser. Cracks were irregularly generated at perpendicular direction to laser axis by balance of internal stress in a large volume of absorption area at $10 \mathrm{ps}$ pulsed laser.

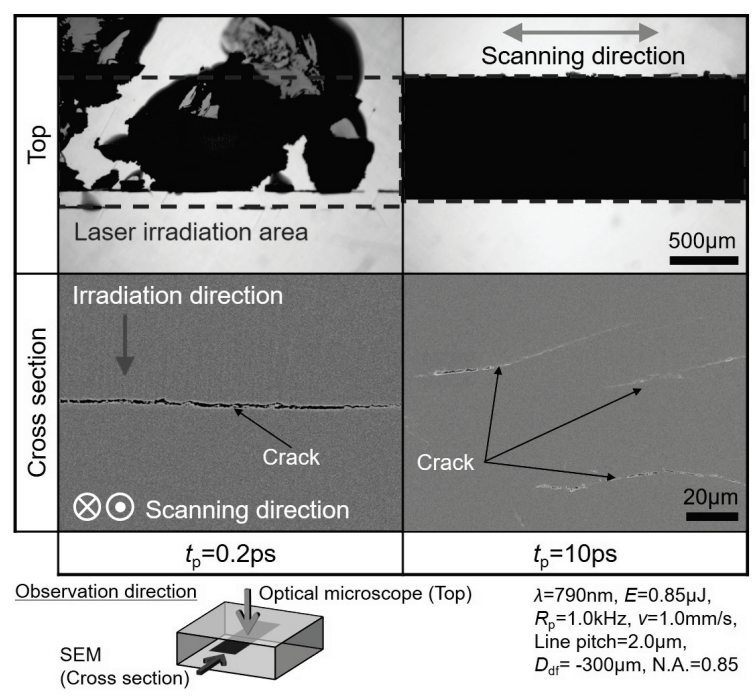

Fig. 4 Optical microphotographs and SEM images of laser irradiation area at $0.2 \mathrm{ps}$ and $10 \mathrm{ps}$

Figure 5 shows optical microphotographs of laser irradiation area created by different line pitches of $2.0 \mu \mathrm{m}$ and $5.0 \mu \mathrm{m}$, and effect of line pitch on internal modified layer thickness was investigated at 10 ps pulse duration. These microphotographs are top and cross sectional views. The continuity of internal modified layer became low at line pitch of $5.0 \mu \mathrm{m}$ compared with $2.0 \mu \mathrm{m}$ line pitch, but almost continuous modified layer could be formed at both line pitches. Although both line pitches showed similar width of internal modified layer, the internal modified layer at line pitch of $5.0 \mu \mathrm{m}$ became thinner than that at line pitch of $2.0 \mu \mathrm{m}$. The thickness of internal modified layer was approximately $40 \mu \mathrm{m}$ at line pitch of $2.0 \mu \mathrm{m}$.

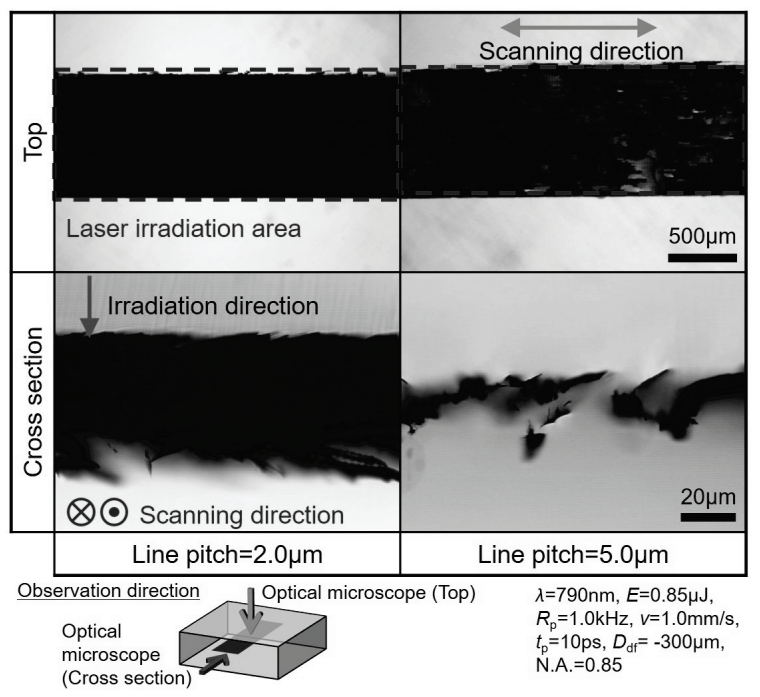

Fig. 5 Optical microphotographs of laser irradiation area at $2.0 \mu \mathrm{m}$ and $5.0 \mu \mathrm{m}$ line pitch 
Judging from these results, thin and uniform internal modified layer can be obtained under the condition of $5.0 \mu \mathrm{m}$ line pitch and $10 \mathrm{ps}$ pulse duration, and the separation of $\mathrm{GaN}$ was conducted in breaking test.

\section{SEPARATION OF GaN BY BREAKING TEST}

Next, separation method of GaN was investigated by using the internal modified layer. Firstly, internal modified layer was formed in $\mathrm{GaN}$ under the condition mentioned above (Pulse duration $10 \mathrm{ps,}$ Line pitch $5.0 \mu \mathrm{m}$ ), as shown in Fig. 6. Then, GaN with internal modified layer was cut by laser scribing according to the size of the laser irradiation area (4 $\mathrm{mm} \times 9 \mathrm{~mm}$ ). In the scribing process, the same laser irradiation condition was used except for focusing position, which was set on the top surface of specimen.

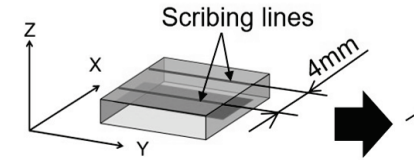

(a) Before scribing

\section{Internal modified layer}

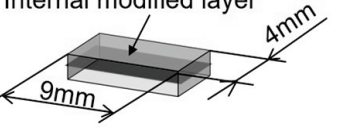

(b) After scribing
Fig. 6 Schematic illustration of scribing process

Next, the top and the bottom surfaces of $\mathrm{GaN}$ were adhered on aluminum plates by using epoxy resin, as shown in Fig. 7. After that, breaking tests were carried out by adding pulling load in perpendicular direction to the internal modified layer. The speed of the crosshead was set to $0.5 \mathrm{~mm} / \mathrm{min}$. Vertical pulling stress was calculated by dividing the maximum load by the area of internal modified layer. The vertical pulling stress was recorded as a breaking stress. GaN was successfully separated by small breaking stress of $670 \mathrm{kPa}$, which is much smaller than ideal strength of approximately $30 \mathrm{GPa}$ calculated by density functional theory ${ }^{15)}$.

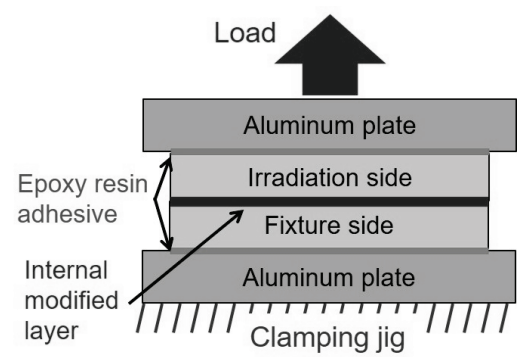

Fig. 7 Schematic illustration of breaking test with perpendicular load to internal modified layer

Figure 8 shows SEM images of the fractured surface at irradiation and fixture sides shown in Fig. 7. Upper images of fractured surface are low magnified view, and the areas of (a) and (b) are magnified in lower images. In magnified views, brittle fracture areas remained linearly on both sides of irradiation and fixture, and these lines are the marks of laser scanning. The interval of these lines is $5.0 \mu \mathrm{m}$, which is corresponded to setting value of $5.0 \mu \mathrm{m}$ line pitch. In addition, circular marks were observed at the fractured surface, and the direction of circular marks became opposite in each scanning line. Because scanning direction was reciprocally changed, as shown in Fig. 2. Thus, these results show that crack was propagated like disk shape, and it is considered that separation of $\mathrm{GaN}$ was successfully started from this internal modified layer. On the other hand, smooth surface area without brittle fracture could be observed in upper area of fractured surface images, and it seemed to resolidified material.

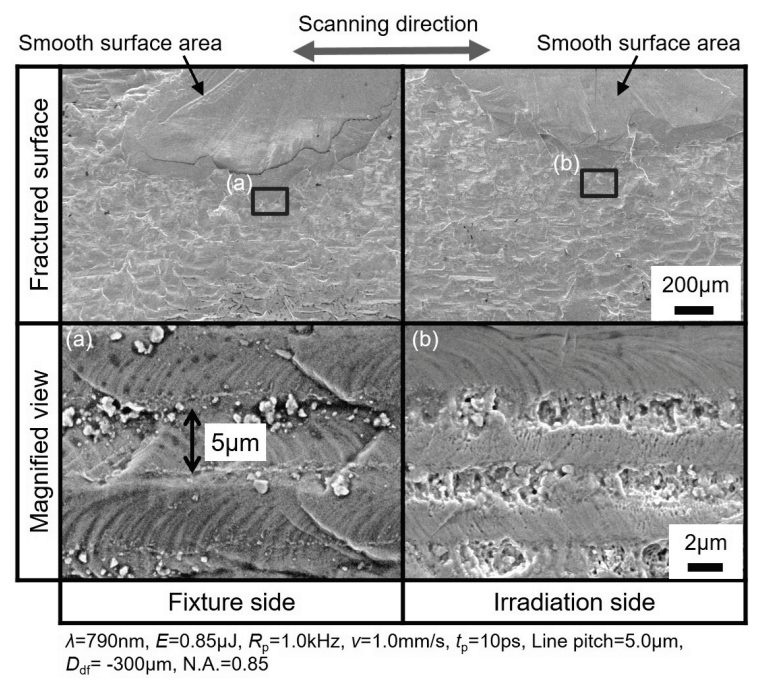

Fig. 8 SEM images of fractured surface at 10 ps

Element analysis of fractured surface was carried out by using energy dispersive X-ray spectrometry (EDS), as shown in Fig 9. Smooth surface areas without brittle fracture shown in Fig. 8 were observed as white islands in SEM image, and color was silver metallic in optical microscopic observation. In white areas of SEM image, nitrogen (N) became low intensity, and gallium (Ga) showed high intensity. In addition, oxygen (O) showed a little high intensity. Judging from these results, these white areas as smooth surface without brittle fracture are gallium oxide, and gallium nitride was partly decomposed into gallium and nitrogen by ultrashort pulse laser irradiation. Thus, it is considered that internal modified layer was created by the combination of brittle fracture and decomposition of GaN. 


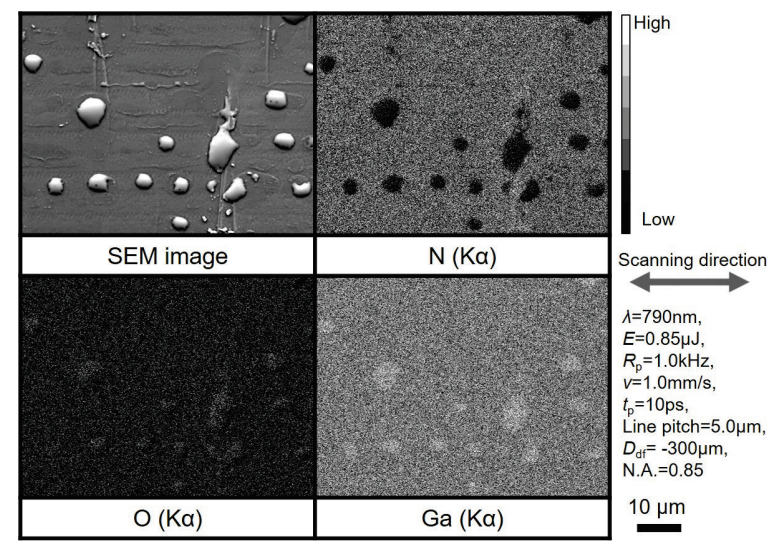

Fig. 9 Elemental analysis of fractured surface at $10 \mathrm{ps}$

Cross section of fractured surface was observed as shown in Fig. 10. Upper images of fractured surface are low magnified view, and the areas of (a) and (b) are magnified in lower images. Almost flat surface could be obtained after the separation of GaN, although there are some concave and convex shapes at the fractured surface. This is because cracks were irregular formed in depth direction at $10 \mathrm{ps}$ as shown in Fig. 4. The surface roughness of these fracture surfaces was approximately $\mathrm{Rz}=45 \mu \mathrm{m}$, which is correspond to the size of internal modified thickness. Kerf loss in this separation method is almost similar to the thickness of internal modified layer, and it is expected to reduce the kerf loss by appropriate control of focusing situation.

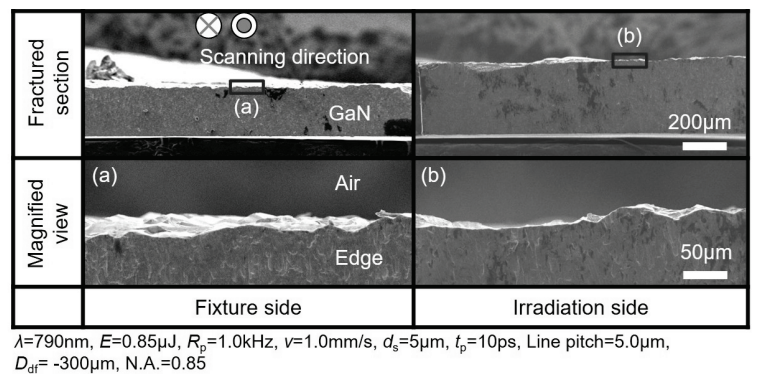

Fig. 10 SEM images in cross sectional view of fractured specimen at $10 \mathrm{ps}$

The refractive index of $\mathrm{GaN}$ is 2.4 , which is very high value compared with glasses. Then, focusing situation inside $\mathrm{GaN}$ becomes loose due to its high spherical aberration, which led to a little thicker internal modified layer with the balance of internal stress in a large volume of absorption area. Further investigation is necessary to obtain thinner internal modified layer by overcoming the focusing situation inside GaN, but the internal modified layer makes it possible to separate GaN by small breaking stress. Ultrashort pulsed laser irradiation of 10 ps can be expected as separation method of gallium nitride at appropriate line pitch.

\section{CONCLUSIONS}

In this study, separation method of gallium nitride was investigated by using internal modified layer formed with ultrashort pulsed laser. The main conclusions obtained in this study are as follows:

(1) Gallium nitride is partly decomposed into gallium and nitrogen by ultrashort pulsed laser irradiation, and internal modified layer is created by the combination of brittle fracture and decomposition of gallium nitride.

(2) Discontinuous internal modified layer with a large crack propagation is created at $0.2 \mathrm{ps}$, while the continuous internal modified layer can be obtained at $10 \mathrm{ps}$ under proper laser intensity conditions.

(3) Appropriate line pitch of laser scanning can form thinner internal modified layer. $5.0 \mu \mathrm{m}$ line pitch makes it possible to form thinner internal modified layer effectively compared with 2.0 $\mu \mathrm{m}$ line pith in the case of $10 \mathrm{ps}$.

(4) Gallium nitride is successfully separated by the continuous internal modified layer with small load.

\section{ACKNOWLEDGMENT}

Authors would like to express their sincere thanks to Dr. Togo Shinonaga, Assistant Professor, Graduate School of Natural Science and Technology, Okayama University for his contribution to the experiments.

\section{REFERENCES}

1) S. Nakamura, T Mukai, M Senoh: High-Power GaN P-N Junction Blue-Light-Emitting Diodes, Japanese Journal of Applied Physics, Vol.30, No.12A, (1991), pp.L1998-L2001.

2) M. Hikita, M. Yanagaihara, Y. Uemoto, T. Ueda, T. Tanaka, D. Ueda: GaN-based Power Devices, Panasonic Technical Journal, Vol.55, No.2, (2009), pp.21-25. (In Japanese)

3) S. Yoshida, H. Ishii, J. Li, D. Wang, M. Ichikawa: A High-power AlGaN/GaN Heterojunction Field-effect Transistor, SolidState Electronics, Vo.47, No.3, (2003), pp.589592.

4) S. Iwakami, M. Yanagihara, O. Machida, E. Chino, N. Kaneko, H. Goto, K. Ohtsuka: AlGaN/GaN Heterostructure Field-effect Transistors (HFETs) on Si Substrates for Large- 
current Operation, Japanese Journal of Applied Physics, Vol.43, No.7A, (2004), pp.L831-L833.

5) H. P. Maruska, J. J. Tietjen: The Preparation and Properties of Vapor-deposited Single-crystalline GaN, Applied Physics Letters. Vol.15, No.10, (1969), pp.327-329.

6) H. Amano, N. Sawaki, I. Akasaki, Y. Toyoda: Metalorganic Vapor Phase Epitaxial Growth of a High Quality GaN Film Using an AlN Buffer Layer, Applied Physics Letters, Vol.48, No.5, (1986), pp.353-355.

7) K. Motoki, T. Okahisa, N. Matsumoto, M. Matsushima, H. Kimura, H. Kasai, K. Takemoto, K. Uematsu, T. Hirano, M. Nakayama, S. Nakahata, M. Ueno, D. Hara, Y. Kumagai, A. Koukitu, H. Seki: Preparation of Large Freestanding GaN Substrates by Hydride Vapor Phase Epitaxy Using GaAs as a Starting Substrate, Japanese Journal of Applied Physics, Vol.40, No.2B, (2001), pp.L140-L143.

8) K. Motoki, T. Okahisa, S. Nakahata, N. Matsumoto, H. Kimura, H. Kasai, K. Takemoto, K. Uematsu, M. Ueno, Y. Kumagai, A. Koukitu, H. Seki: Preparation of Large GaN Substrates, Material Science and Engineering: B, Vol.93, No.1-3, (2002), pp.123-130.

9) K. Motoki, T. Okahisa, S. Nakahata, N. Matsumoto, H. Kimura, H. Kasai, K. Takemoto, K. Uematsu, M. Ueno, Y. Kumagai, A. Koukitu, H. Seki: Growth and Characterization of Freestanding GaN Substrates, Journal of Crystal Growth Vol.237-239, No.2, (2002), pp.912-921.

10) H. Amano: Bulk Single Crystals of Group III Nitride Substrates and Lattice-Matched
Substrates for High Performance Nitride-Based Devices, CMC Publishing Co., Ltd., (2009), p.22. (In Japanese)

11) S. Hirata: New Laser Slicing Technology Named KABRA Process Enables High Speed and High Efficiency SiC Slicing, Proceedings of SPIE 10520, Laser-based Micro- and Nanoprocessing XII, 1052003, (2018), doi: 10.1117/12. 2291458.

12) Y. Yamada, Y. Kaneko, R. Aoki, J. Ikeno, H. Suzuki: Laser Cutting Out Process for Semiconductor Crystal Material Applying Laser Slicing Method, Journal of Japan Society for the Precision Engineering, Vol.83, No.4, (2017), pp.375-380.

13) I. N. Zavestovskaya, N. A. Kozlovskaya, O. N. Krokhin: Laser Technologies for Processing Wide Band-gap Semiconductors and Insulators: Nonlinear Absorption Mechanisms, Bulletin of the Lebedev Physics Institute, Vol.42, No.4, (2015), pp.110-114.

14) M. Sakakura, Y. Ishiguro, Y. Shimotsuma, N. Fukuda, K. Miura: Modulation of Transient Stress Distributions for Controlling Femtosecond Laser-induced Cracks inside a Single Crystal, Applied Physics A: Materials Science and Processing, Vol.114, No.1, (2013), pp.261-265.

15) Y. Umeno, A. Kubo, S. Naga: Density Functional Theory Calculation of Ideal Strength of $\mathrm{SiC}$ and GaN: Effect of Multi-axial Stress, Computational Materials Science, Vol.109, (2015), pp.105-110. 